Supporting Information for:

\title{
MAI Termination Favors Efficient Hole Extraction and Slow Charge Recombination at $\mathrm{MAPbI}_{3} / \mathrm{CuSCN}$ Heterojunction
}

Jinlu He, ${ }^{1}$ David Casanova, ${ }^{2,3}$ Wei-Hai Fang,,${ }^{1}$ Run Long, ${ }^{1 *}$ Oleg V. Prezhdo ${ }^{4}$

${ }^{1}$ College of Chemistry, Key Laboratory of Theoretical \& Computational

Photochemistry of Ministry of Education, Beijing Normal University, Beijing, 100875,

$$
\text { P. R. China }
$$

${ }^{2}$ Kimika Fakultatea, Euskal Herriko Unibertsitatea and Donostia International Physics Center 20018 Donostia, Euskadi, Spain

${ }^{3}$ IKERBASQUE, Basque Foundation for Science, 48013 Bilbao, Euskadi, Spain

${ }^{4}$ Department of Chemistry, University of Southern California, Los Angeles, CA 90089,

$$
\text { United States }
$$

*Corresponding author, E-mail: runlong@bnu.edu.cn 


\section{Tests of Energy Level Alignment and Charge Density Localization for Different}

\section{Simulation Cell Setups.}

As typical of most periodic slab calculations, a vacuum layer separates slab images in the original simulation cell, Figure 1 of the main text, in the direction perpendicular to the $\mathrm{MAPbI}_{3} / \mathrm{CuSCN}$ interface, in order to avoid spurious interactions between slab images. As a result, each material contains a surface facing the vacuum region. It is possible that such surfaces create spurious states, and thicker slabs with a larger bulklike region are desired. Currently, thick slabs cannot be used in ab initio NAMD calculations due to computational expense. Although surface states can influence the dynamics, they contribute little to the coupling across the interface where both charge separation and recombination dynamics take place. An alternative setup, with two interfaces and no vacuum can also be used, but it carries own drawbacks in NAMD calculations. Interfacial states become quasi doubly degenerate at the two interfaces. Charges can artificially hop between such states, introducing significant errors to NA couplings and decoherence times. Both types of setup, including up to 942 atoms, are tested in the figures below.

The conclusions of the present work are independent of the setup, because they are based on qualitative arguments that are invariant to the simulation cell details. Because the valence band (VB) maximum of $\mathrm{MAPbI}_{3}$ originates from iodine orbitals, while the conduction band $(\mathrm{CB})$ minimum arises from $\mathrm{Pb}$ orbitals, MAI termination places $\mathrm{VB}$ states close to $\mathrm{CuSCN}$, favoring hole extraction from $\mathrm{MAPbI}_{3}$. At the same time MAI termination moves $\mathrm{CB}$ states away from $\mathrm{CuSCN}$, creating an MAI barrier between electrons in $\mathrm{MAPbI}_{3}$ and holes in $\mathrm{CuSCN}$, and slowing down electron-hole recombination. Both factors improve the solar cell performance. The opposite is true of the $\mathrm{PbI}_{2}$ termination, which deteriorates the performance. 

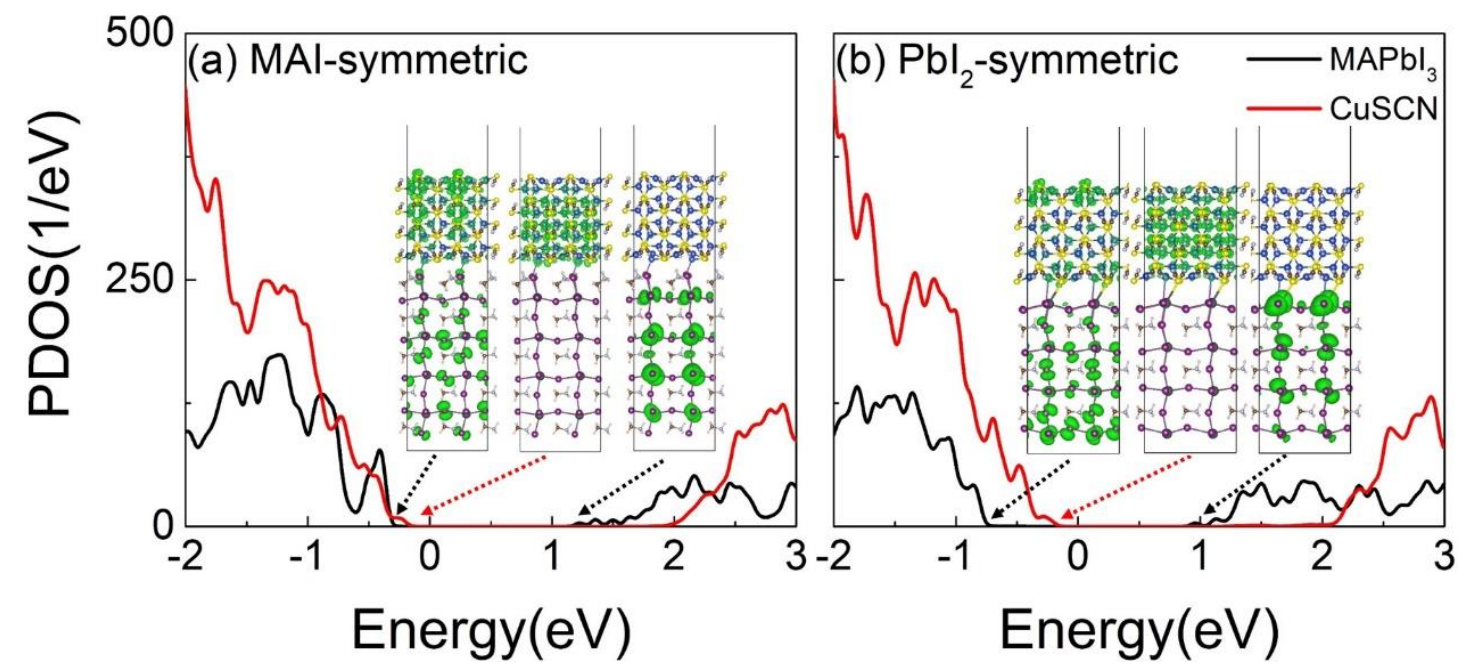

Figure S1. Projected density of states (PDOS) of $\mathrm{MAPbI}_{3} / \mathrm{CuSCN}$ heterojunctions with (a) MAI- and (b) $\mathrm{PbI}_{2}$-terminated interfaces and symmetric $\mathrm{MAPbI}_{3}$ slab. Charge densities of the key orbitals involved in the hole transfer and electron-hole recombination processes are shown in the insets. Zero energy is set to the Fermi level corresponding to the valence band maximum of the system. The symmetric 324-atom MAI-terminated and 234-atom $\mathrm{PbI}_{2}$-terminated $(2 \times 3)(001) \mathrm{MAPbI}_{3}$ surfaces are interfaced with the 256-atom $\mathrm{CuSCN}(2 \times 2)(010)$ surface. Holes in $\mathrm{MAPbI}_{3}$ are supported by iodine atoms. Direct contact of iodines with $\mathrm{CuSCN}$, as well as participation of high frequency MA motions, facilitate rapid hole transfer from $\mathrm{MAPbI}_{3}$ to $\mathrm{CuSCN}$ for the MAI termination. Electrons in $\mathrm{MAPbI}_{3}$ are supported by $\mathrm{Pb}$ atoms. Spatial separation of the $\mathrm{MAPbI}_{3}$ electrons from the $\mathrm{CuSCN}$ holes at the MAIterminated interface favors slow charge recombination. 

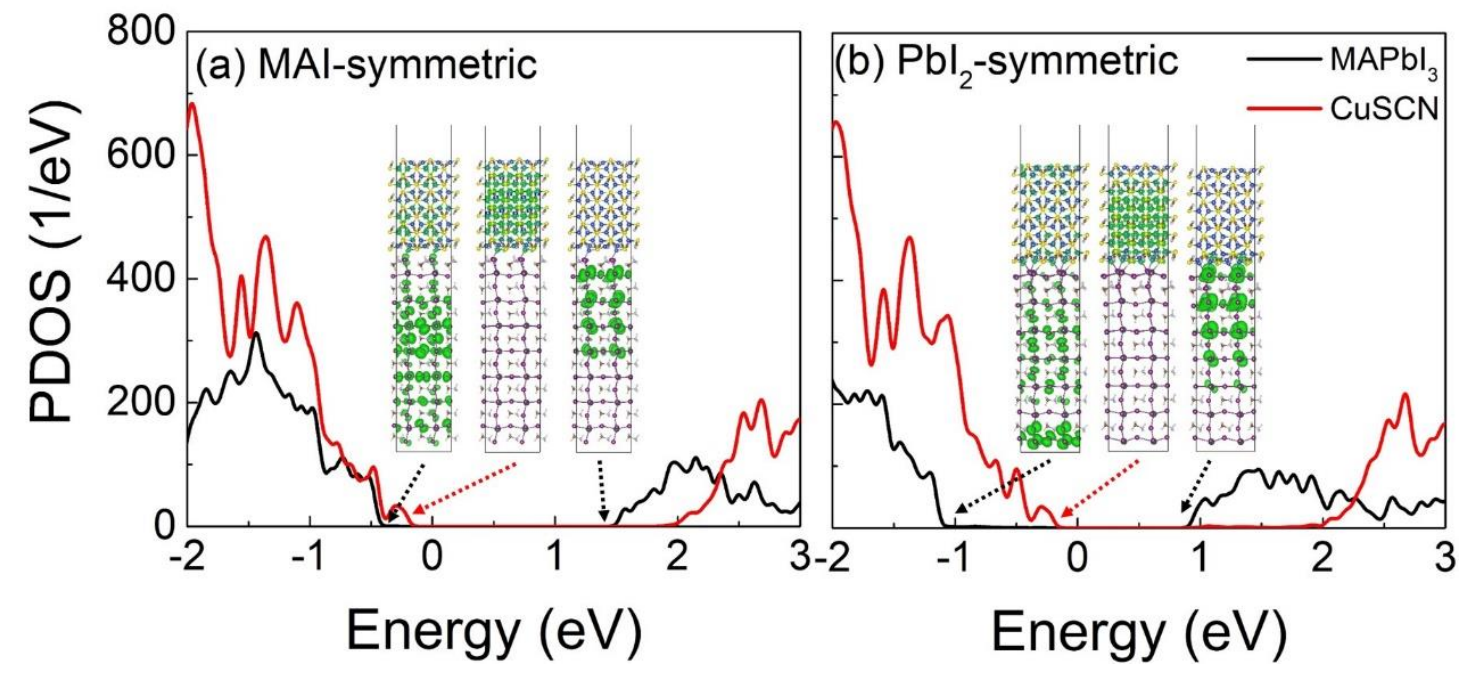

Figure S2. Projected density of states (PDOS) of $\mathrm{MAPbI}_{3} / \mathrm{CuSCN}$ heterojunctions with (a) MAI- and (b) $\mathrm{PbI}_{2}$-terminated interfaces and symmetric $\mathrm{MAPbI}_{3}$ slab. Charge densities of the key orbitals involved in the hole transfer and electron-hole recombination processes are shown in the insets. Zero energy is set to the Fermi level corresponding to the valence band maximum of the system. The symmetric 558-atom MAI-terminated and 450-atom $\mathrm{PbI}_{2}$-terminated $(2 \times 3)(001) \mathrm{MAPbI}_{3}$ surfaces are interfaced with the 384-atom CuSCN $(2 \times 2)(010)$ surface. Holes in $\mathrm{MAPbI}_{3}$ are supported by iodine atoms. Direct contact of iodines with $\mathrm{CuSCN}$, as well as participation of high frequency MA motions, facilitate rapid hole transfer from $\mathrm{MAPbI}_{3}$ to $\mathrm{CuSCN}$ for the MAI termination. Electrons in $\mathrm{MAPbI}_{3}$ are supported by $\mathrm{Pb}$ atoms. Spatial separation of the $\mathrm{MAPbI}_{3}$ electrons from the $\mathrm{CuSCN}$ holes at the MAIterminated interface favors slow charge recombination. 

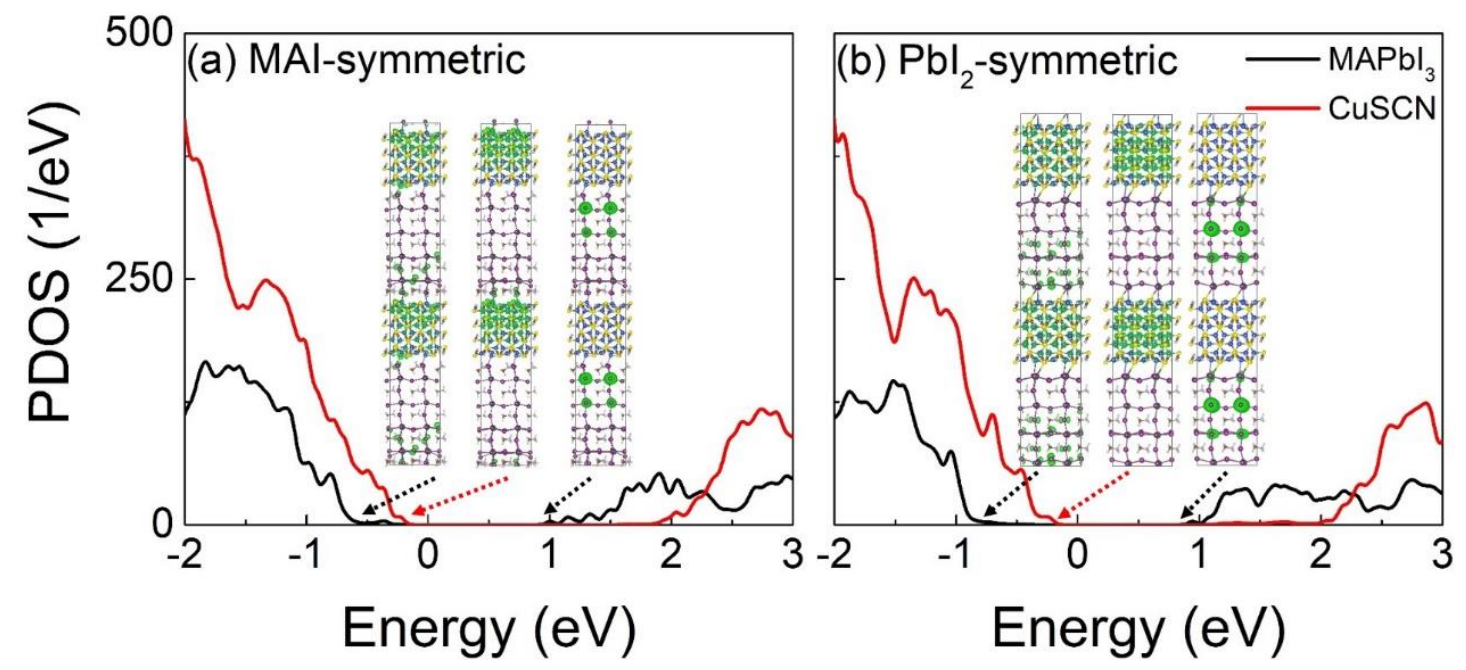

Figure S3. Projected density of states (PDOS) of $\mathrm{MAPbI}_{3} / \mathrm{CuSCN}$ heterojunctions with (a) MAI- and (b) $\mathrm{PbI}_{2}$-terminated interfaces and symmetric $\mathrm{MAPbI}_{3}$ slab by removing the vacuum form the simulation cells shown in Figure S1. Charge densities of the key orbitals involved in the hole transfer and electron-hole recombination processes are shown in the insets. Zero energy is set to the Fermi level corresponding to the valence band maximum of the system. Holes in $\mathrm{MAPbI}_{3}$ are supported by iodine atoms. Direct contact of iodines with $\mathrm{CuSCN}$, as well as participation of high frequency MA motions, facilitate rapid hole transfer from $\mathrm{MAPbI}_{3}$ to $\mathrm{CuSCN}$ for the MAI termination. Electrons in $\mathrm{MAPbI}_{3}$ are supported by $\mathrm{Pb}$ atoms. Spatial separation of the $\mathrm{MAPbI}_{3}$ electrons from the CuSCN holes at the MAI-terminated interface favors slow charge recombination. 


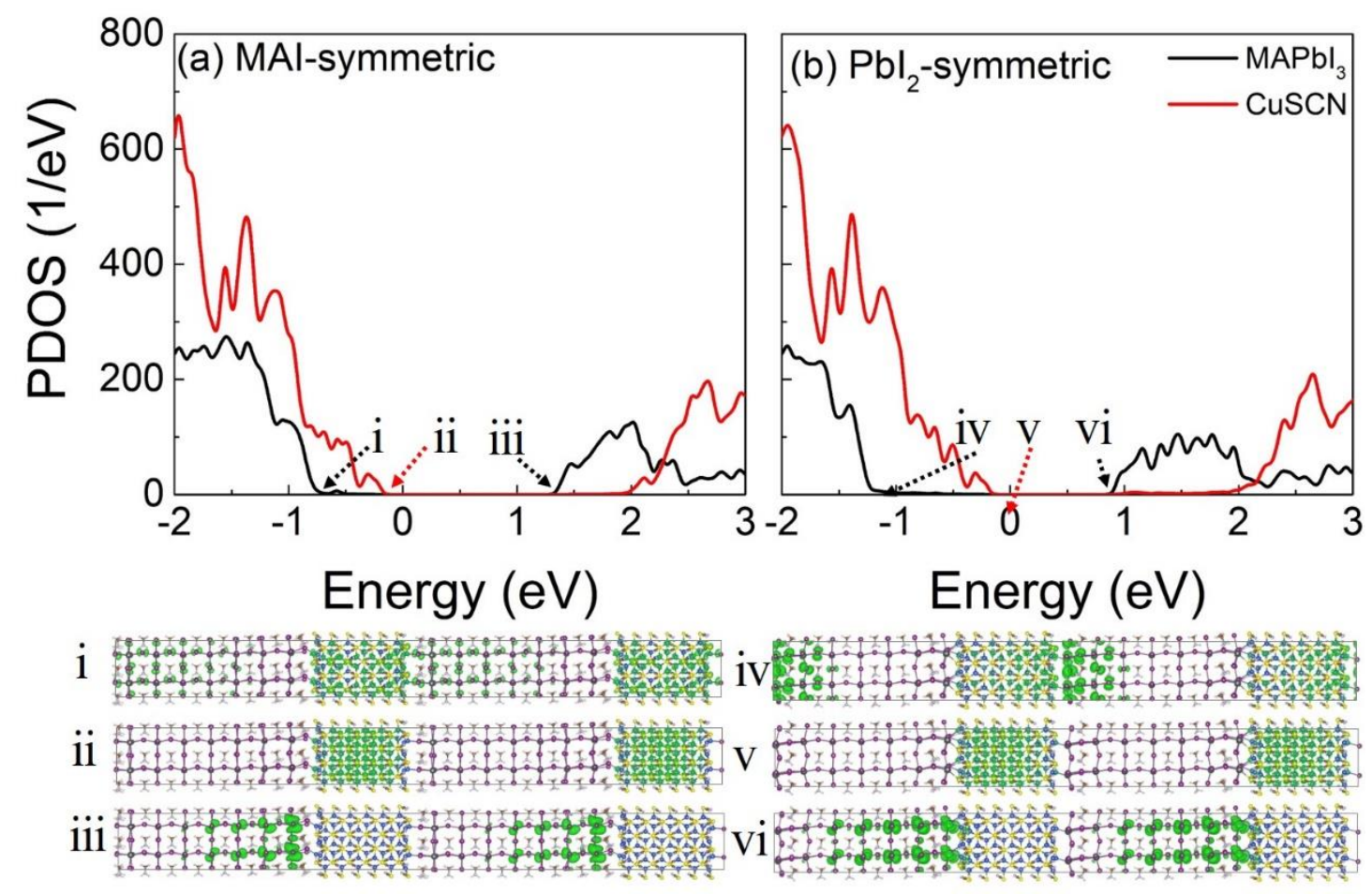

Figure S4. Projected density of states (PDOS) of $\mathrm{MAPbI}_{3} / \mathrm{CuSCN}$ heterojunctions with (a) MAI- and (b) $\mathrm{PbI}_{2}$-terminated interfaces and symmetric $\mathrm{MAPbI}_{3}$ slab by removing the vacuum form the simulation cells shown in Figure S2. Charge densities of the key orbitals involved in the hole transfer and electron-hole recombination processes are shown below (a) and (b). Zero energy is set to the Fermi level corresponding to the valence band maximum of the system. Holes in $\mathrm{MAPbI}_{3}$ are supported by iodine atoms. Direct contact of iodines with $\mathrm{CuSCN}$, as well as participation of high frequency MA motions, facilitate rapid hole transfer from $\mathrm{MAPbI}_{3}$ to $\mathrm{CuSCN}$ for the MAI termination. Electrons in $\mathrm{MAPbI}_{3}$ are supported by $\mathrm{Pb}$ atoms. Spatial separation of the $\mathrm{MAPbI}_{3}$ electrons from the CuSCN holes at the MAI-terminated interface favors slow charge recombination. 
Table S1. Ensemble Averaged Absolute Values of Nonadiabatic Couplings, in meV, between Adjacent VB States in the MAI- and $\mathrm{PbI}_{2}$-terminated $\mathrm{MAPbI}_{3} / \mathrm{CuSCN}$ Heterojunctions.

\begin{tabular}{|c|c|c|}
\hline & MAI-terminated & PbI $_{2}$-terminated \\
\hline VBM-10/VBM-9 & 88.35 & 55.98 \\
\hline VBM-9/VBM-8 & 84.92 & 61.67 \\
\hline VBM-8/VBM-7 & 84.32 & 56.98 \\
\hline VBM-7/VBM-6 & 91.05 & 53.12 \\
\hline VBM-6/VBM-5 & 88.16 & 52.87 \\
\hline VBM-5/VBM-4 & 83.22 & 53.64 \\
\hline VBM-4/VBM-3 & 80.28 & 50.07 \\
\hline VBM-3/VBM-2 & 81.81 & 52.61 \\
\hline VBM-2/VBM-1 & 84.05 & 54.49 \\
\hline VBM-1/VBM & 83.14 & 50.49 \\
\hline
\end{tabular}

